10. Lu L, Lin H, Tian L, Yang W, Sun J, Liu Q. Time series analysis of dengue fever and weather in Guangzhou, China. BMC Public Health. 2009;9:395. http://dx.doi.org/10.1186/1471-2458-9-395

Address for correspondence: Dexin Li, National Institute for Viral Disease Control and Prevention, Chinese Center for Disease Control and Prevention, Changbai Rd 155, Changping District, Beijing 102206, China; email: lidx@chinacdc.cn; or Guoliang Hu, Jiangxi Province Center for Disease Control and Prevention, Beijingdong Rd 555, Nanchang 330029, China; email: jxcdchg1@163.com

\title{
Pericarditis Caused by Hyperinvasive Strain of Neisseria meningitidis, Sardinia, Italy, 2015
}

\section{Cecilia Fazio, Paolo Castiglia, Andrea Piana, Arianna Neri, Maria S. Mura, Giorgia Caruana, Paola Vacca, Anna Anselmo, Andrea Ciammaruconi, Antonella Fortunato, Anna M. Palozzi, Silvia Fillo, Florigio Lista, Paola Stefanelli}

Author affiliations: Istituto Superiore di Sanità, Rome, Italy (C. Fazio, A. Neri, P. Vacca, P. Stefanelli); Università di Sassari, Sassari, Italy (P. Castiglia, A. Piana, M.S. Mura, G. Caruana); Centro Studi e Ricerche di Sanità e Veterinaria dell'Esercito, Rome (A. Anselmo, A. Ciammaruconi, A. Fortunato, A.M. Palozzi, S. Fillo, F. Lista)

\section{DOI: http://dx.doi.org/10.3201/eid2206.160160}

To the Editor: Invasive meningococcal disease is usually defined by the occurrence of meningitis or septicemia. Pericarditis might occur during the course of invasive infection. This clinical picture, defined as disseminated meningococcal disease with pericarditis (1) or secondary meningococcal pericarditis, was reported in 1918 (2). In 1939, primary or isolated meningococcal pericarditis $(1,3)$ was described. In this form of pericarditis, pericardial or blood cultures are positive for Neisseria meningitidis but there is no meningeal involvement or clinical meningococcemia (4).

Since its description, several cases of primary meningococcal pericarditis have been reported (5). Although its pathogenesis remains largely undefined, it has been hypothesized that the onset of primary pericarditis occurs after a transient bacteremia or as a consequence of involvement of the lower respiratory tract (4). Blaser et al. reported that serogroup $\mathrm{C}$ meningococci are usually associated with this disease, especially in adults. However, serogroups B,
$\mathrm{W}$, and $\mathrm{Y}$ have also been identified (4). We report a casepatient with primary meningococcal pericarditis caused by a serogroup $\mathrm{C}$ strain of $N$. meningitidis.

The patient was a 32-year-old man who lived in Sardinia, Italy. He had no predisposing factors, such as immunodeficiency or other chronic disorders. Disease onset occurred on August 29, 2015. Clinical manifestations were fever (temperature $38^{\circ} \mathrm{C}$ ), hypotension, epigastralgia, arthralgia, asthenia, chest pain, and reduced vesicular murmur. The left ventricle was widely hypokinetic, and a light ST increase was observed. A blood culture was positive for N. meningitidis.

The patient was given piperacillin/tazobactam $(4.5 \mathrm{~g}$ $3 \times / \mathrm{d})$ and metronidazole $(500 \mathrm{mg} 3 \times / \mathrm{d})$ for 4 days. After 4 days, treatment with ceftriaxone $(2 \mathrm{~g} 2 \times / \mathrm{d})$ for 4 days was started. Because of persistent fever $\left(38.8^{\circ} \mathrm{C}\right)$, levofloxacin (500 mg $2 \times / d$ ) for 23 days was also started on day 7 . On day 10 , ceftriaxone was replaced with piperacillin/tazobac$\operatorname{tam}(4.5 \mathrm{~g} 4 \times / \mathrm{d})$ for 21 days. A major bilateral pleural effusion was detected on the left side. On day 11, the fever had resolved. The outcome was favorable for this patient.

Drug resistance of the strain was determined by using the MIC Test Strip Method (Liofilchem, Abruzzi, Italy). Breakpoints used were those recommended by the European Committee on Antimicrobial Susceptibility Testing version 5.0 (http://www.eucast.org/). The strain was susceptible to cefotaxime, ceftriaxone, ciprofloxacin, penicillin $\mathrm{G}$, and rifampin. Serogroup was determined by using slide agglutination with commercial antisera (Remel Europe, Ltd., Dartford, UK) and confirmed by PCR ( 6 ).

Whole-genome sequencing was conducted to obtain molecular data and enable comparison with other meningococci of the same serogroup that were isolated in Italy. Multilocus sequence typing (MLST) and typing of porA and fetA genes and Bexsero (meningococcal group B vaccine) antigen genes (http://www.fda.gov/Biologics BloodVaccines/Vaccines/ApprovedProducts/ucm431374. $\mathrm{htm}$ ) were conducted as described (http://neisseria.org/). Whole-genome sequence was analyzed by using the BIGSdb Genome Comparator Tool (http://pubmlst.org/ neisseria/). Genomes of meningococci belonging to the same finetype were compared by using the core genome MLST (cgMLST) approach.

The $N$. meningitidis strain of serogroup C was susceptible to all antimicrobial drugs tested. Although serogroup $\mathrm{C}$ was associated with 53 (41\%) of 132 invasive meningococcal disease cases in Italy in 2015 (http://www.iss.it/ binary/mabi/cont/Report_MBI_20151223_v4.pdf), this serogroup has not been detected in Sardinia since 2010.

Molecular analyses showed that the strain belonged to the hypervirulent clonal complex (cc) 11, sequence type (ST) 11. The complete finetype was C:5-1,10-8:F3-6:ST11(cc11). This finetype has been reported in the United 
States and several countries in Europe (7), including Italy, and is responsible for several disease outbreaks. In Italy, this finetype represents $61 \%(70 / 115)$ of all serogroup C strains collected during 2012-2015. Two outbreaks caused by this strain were reported in Italy in 2007 (8) and in 2012 (9). The $N$. meningitidis factor $\mathrm{H}$ binding protein and heparin binding protein alleles were 1.13 and 20, respectively. The $N$. meningitidis adhesin A variant had an insertion sequence that disrupted this gene, as described for ET-15 meningococci (10). On the basis of results of cgMLST, the strain was determined to be related to strains responsible for an outbreak in Italy in 2015.

In summary, we report a case of meningococcal pericarditis caused by a strain of $N$. meningitidis. This strain belongs to hyperinvasive clonal complex cc11 and was identified as C:P1.5-1,10-8:F3-6:ST-11(cc11), an emerging strain in Italy and worldwide. Timely diagnosis and complete molecular characterization of this strain, which causes a rare form of invasive disease (4), is needed for appropriate management of patients with this disease.

\section{Acknowledgments}

We thank Luigina Ambrosio for technical assistance. We used the Neisseria Multi Locus Sequence Typing website developed by Keith Jolley and sited at the University of Oxford (Oxford, UK).

Collection of invasive meningococcal isolates within the National Surveillance System of Invasive Meningococcal Disease was supported by a Ministry of Health-National Center for Disease Prevention and Control Project (Disease Surveillance of Invasive Neisseria meningitidis, Streptococcus pneumoniae, and Haemophilus influenza, 2014). Development of the Neisseria Multi Locus Sequence Typing website has been supported by the Wellcome Trust and European Union.

\section{References}

1. Finkelstein Y, Adler Y, Nussinovitch M, Varsano I. A new classification for pericarditis associated with meningococcal infection. Eur J Pediatr. 1997;156:585-8. http://dx.doi.org/10.1007/ s004310050669

2. Herrick WW. Meningococcic pericarditis, with report of 12 cases: medical service of the base hospital, Camp Jackson, S.C. Med Clin North Am. 1918;2:411-26.

3. Orgain ES, Postom MA. Pericarditis with effusion due to meningococcus. Am Heart J. 1939;18:368. http://dx.doi.org/ 10.1016/S0002-8703(39)90637-X

4. Blaser MJ, Reingold AI, Alsever RN, Hightower A. Primary meningococcal pericarditis: a disease of adults associated with serogroup C Neisseria meningitidis. Rev Infect Dis. 1984;6:62532. http://dx.doi.org/10.1093/clinids/6.5.625

5. Nkosi J, Thakrar A, Kumar K, Ahmadie R, Fang T, Lytwyn M, et al. Meningococcal serotype Y myopericarditis. Diagn Microbiol Infect Dis. 2009;63:223-7. http://dx.doi.org/10.1016/ j.diagmicrobio.2008.09.015

6. Zhu H, Wang Q, Wen L, Xu J, Shao Z, Chen M, et al. Development of a multiplex PCR assay for detection and genogrouping of
Neisseria meningitidis. J Clin Microbiol. 2012;50:46-51. http://dx.doi.org/10.1128/JCM.00918-11

7. Aubert L, Taha MK, Boo N, Le Strat Y, Deghamane AE, Sanna A, et al. Serogroup $\mathrm{C}$ invasive meningococcal disease among men who have sex with men and in gay-oriented social venues in the Paris region: July 2013 to December 2014. Euro Surveill. 2015;20:pii: 21016.. http://dx.doi.org/10.2807/1560-7917. ES2015.20.3.21016

8. Fazio C, Neri A, Tonino S, Carannante A, Caporali MG, Salmaso S, et al. Characterization of Neisseria meningitidis $\mathrm{C}$ strains causing two clusters in the north of Italy in 2007 and 2008. Euro Surveill. 2009;14:pii: 19179.

9. Stefanelli P, Fazio C, Neri A, Isola P, Sani S, Marelli P, et al. Cluster of invasive Neisseria meningitidis infections on a cruise ship, Italy, October 2012. Euro Surveill. 2012;17:pii: 20336.

10. Elias J, Vogel U. IS1301 fingerprint analysis of Neisseria meningitidis strains belonging to the ET-15 clone. J Clin Microbiol. 2007;45:159-67. http://dx.doi.org/10.1128/JCM.01322-06

Address for correspondence: Paola Stefanelli, Department of Infectious, Parasitic and Immuno-Mediated Diseases, Istituto Superiore di Sanità, Viale Regina Elena 299, Rome, Italy; email: paola.stefanelli@iss.it

\section{Ecologic Study of Meningococcal B Vaccine and Neisseria gonorrhoeae Infection, Norway}

\section{Jane Whelan, Hilde Kløvstad, Inger Lise Haugen, Mirna Robert-Du Ry van Beest Holle, Jann Storsaeter}

Author affiliations: Novartis Pharma BV, ${ }^{1}$ Amsterdam, the Netherlands (J. Whelan, M. Robert-Du Ry van Beest Holle); Norwegian Institute of Public Health, Oslo, Norway (H. Kølvstad, I.L. Haugen, J. Storsaeter)

DOI: http://dx.doi.org/10.3201/eid2206.151093

To the Editor: Gonorrhea is a sexually transmitted disease that can cause pelvic inflammatory disease, ectopic pregnancy, and salpingitis in women and infertility in men and women. Rates vary; incidence is 12.5 cases $/ 100,000$ population in Europe $(1)$ and $\approx 6,000$ cases/100,000 population in parts of sub-Saharan Africa (2). Recurrent infection is common, antimicrobial drug resistance is growing, and no licensed vaccine is available to protect against gonorrhea infection. Components of some meningococcal B (MenB) vaccines could provide protection against the causative bacterium, Neisseria gonorrhoeae (M. Pizza, pers. comm.), because the meningococcus bacterium is of the same Neisseria genus and the 2 bacteria share key protein antigens, such as the outer membrane vesicle (OMV). Ecologic evidence from Cuba supports a decline in gonococcus

${ }^{1}$ Now a GlaxoSmithKline company. 